\title{
ARTICLE
}

Received 23 Sep 2014 | Accepted 10 Feb 2015 | Published 16 Mar 2015

DOI: $10.1038 /$ ncomms 7593

\section{Topological crystalline metal in orthorhombic perovskite iridates}

Yige Chen ${ }^{1}$, Yuan-Ming Lu ${ }^{2,3} \&$ Hae-Young Kee $\mathrm{K}^{1,4}$

Since topological insulators were theoretically predicted and experimentally observed in semiconductors with strong spin-orbit coupling, increasing attention has been drawn to topological materials that host exotic surface states. These surface excitations are stable against perturbations since they are protected by global or spatial/lattice symmetries. Following the success in achieving various topological insulators, a tempting challenge now is to search for metallic materials with novel topological properties. Here we predict that orthorhombic perovskite iridates realize a new class of metals dubbed topological crystalline metals, which support zero-energy surface states protected by certain lattice symmetry. These surface states can be probed by photoemission and tunnelling experiments. Furthermore, we show that by applying magnetic fields, the topological crystalline metal can be driven into other topological metallic phases, with different topological properties and surface states.

\footnotetext{
${ }^{1}$ Department of Physics, University of Toronto, Toronto, Ontario M5S 1A7, Canada. ${ }^{2}$ Department of Physics, University of California, Berkeley, California 94720 USA. ${ }^{3}$ Materials Sciences Division, Lawrence Berkeley National Laboratory, Berkeley, California 94720, USA. ${ }^{4}$ Canadian Institute for Advanced Research, Toronto, Ontario M5G 1Z8, Canada. Correspondence and requests for materials should be addressed to H.-Y.K. (email: hykee@physics.utoronto.ca).
} 
R ecent discovery of topological insulators (TIs) reveals a large class of new materials that, despite an insulating bulk, host robust metallic surface states ${ }^{1-9}$. Unlike conventional ordered phases characterized by their symmetries, these quantum phases are featured by non-trivial topology of their band structures, and remarkably they harbour conducting surface states protected by global symmetries such as time-reversal and charge conservation. More recently, it was realized that certain insulators can support surface states protected by crystal symmetry, and they are named topological crystalline insulators ${ }^{10-12}$. The rich topology of insulators in the presence of symmetries lead to a natural question: are there similar 'topological metals' hosting protected surface states? After the proposal of Weyl semimetal ${ }^{13}$, a large class of topological metals are classified ${ }^{14}$, which harbours surface flat bands protected by global symmetries such as charge conservation. However, an experimental confirmation of these phases is still lacking ${ }^{15}$.

In this work, we propose that orthorhombic perovskite iridates $\mathrm{AIrO}_{3}$, where $\mathrm{A}$ is an alkaline-earth metal, with strong spin-orbit coupling (SOC) and Pbnm structure, can realize a new class of metal dubbed topological crystalline metal (TCM). Topological properties of such a TCM phase include zero-energy surface states protected by the mirror-reflection symmetry, and gapless helical modes located at the core of lattice dislocations. Photoemission and tunnelling spectroscopy are natural experimental probes for these topological surface states. We further show how this TCM phase can be driven to metallic phases with different topology by applying magnetic fields, which breaks the mirror symmetry. All these results will be supported by topological classification in the framework of K-theory, as well as numerical calculations of topological invariants and surface/dislocation spectra, as presented in the Methods section.

\section{Results}

Crystal structure and lattice symmetry in $\mathrm{SrIrO}_{3}$. Iridates have attracted much attention due to the strong SOC in 5d-Iridium (Ir) and a variety of crystal structures ranging from layered perovskites to pyrochlore lattices ${ }^{16,17}$. Despite structural differences, a common ingredient of these iridates with $\mathrm{Ir}^{4+}$ is $J_{\text {eff }}=1 / 2$ states governing low-energy physics, resulted from a combination of strong SOC and crystal field splitting. Among them, orthorhombic perovskite iridates $\mathrm{AIrO}_{3}$ (where $\mathrm{A}$ is an alkaline-earth metal) belongs to $\mathrm{Pbnm}$ space group and can be tuned into a $\mathrm{TI}^{18}$.

A unit cell of $\mathrm{AIrO}_{3}$ contains four Ir atoms as shown in Fig. 1a, and there are three types of symmetry plane: b-glide, $n$-glide and mirror plane perpendicular to $\hat{\mathbf{c}}$ axis. Each of them can be assigned to the symmetry operators as follows: $\Pi_{b}, \Pi_{n}$ and $\Pi_{m}$, as listed in the Methods section. Introducing three Pauli matrices corresponding to the in-plane sublattice $(\tau)$, layer $(\boldsymbol{v})$ and pseudo-spin $J_{\text {eff }}=1 / 2(\boldsymbol{\sigma})$, the tight-binding Hamiltonian $H(\mathbf{k})^{18}$ has a relatively simple form shown in equation (6).

The band structure of tight-binding Hamiltonian $H(\mathbf{k})$ exhibits a ring-shaped one-dimensional (1D) Fermi surface (FS) close to the Fermi level as shown in Fig. 1b, which we call the nodal ring, as the energy dispersion is linear in two perpendicular directions. This nodal ring FS was confirmed by first-principle $a b$ initio calculations $^{18,19}$, and it remains intact in the presence of Hubbard $U$ up to $2.5 \mathrm{eV}$. Therefore, the semimetallic character of $\mathrm{SrIrO}_{3}$ with $U$ around $2 \mathrm{eV}$ is consistent with the previous experimental results $^{20,21}$. It was further shown that the size of nodal ring is determined by rotation and tilting angles of the oxygen octahedra around each $\mathrm{Ir}$ atom. In the case of $\mathrm{SrIrO}_{3}$, which has a minimal distortion of octahedra, the nodal ring is centred around the $U \equiv$ $\left(k_{a}=0, k_{b}=\frac{\pi}{a}, k_{c}=\frac{\pi}{c}\right)$ point, and extends in two-dimensional (2D) U-R-S-X plane (perpendicular to $\hat{\mathbf{b}}$ axis) in threedimensional (3D) Brillouin zone (BZ) as shown in Fig. $1 \mathrm{~b}$.

Zero-energy surface states and dislocation helical modes. Here we show that the nodal ring FS exhibits non-trivial topology that leads to localized surface zero modes protected by the mirror symmetry, thus coined TCM. To demonstrate the existence of zero-energy surface states, the band structure calculation for the âc-side plane was carried out with open boundary, that is, $[1 \overline{1} 0]$ surface perpendicular to $\hat{\mathbf{b}}$ axis. The energy dispersion at $k_{c}=\frac{\pi}{c}$, as displayed in Fig. 1c, reveals a dispersionless zero-energy flat band marked by red colour for all $k_{a}$ on [1 $\left.1 \overline{1} 0\right]$ surface of the sample. On the other hand, the slab spectrum at $k_{a}=0$ shows that the surface states are gapped except at $k_{c}=\frac{\pi}{c}$, as shown in Fig. 1d. Similar calculations for $\hat{\mathbf{b}} \hat{\mathbf{c}}$-side plane show that [110] surface (perpendicular to $\hat{\mathbf{a}}$ axis) also supports localized surface zero modes at $k_{c}=\frac{\pi}{c}$, while [010] surface (perpendicular to $\hat{\mathbf{y}}$ axis) does not harbour any zero-energy states. As elaborated in the Methods section, these surface states manifest a mirror-symmetry-protected weak index labelled by a vector ${ }^{22} \mathbf{M}=\hat{\mathbf{a}}+\hat{\mathbf{b}} / / \hat{\mathbf{y}}$, where $\hat{\mathbf{a}}$ and $\hat{\mathbf{b}}$ are Bravais lattice primitive vectors. A direct consequence of this weak index is the existence of $k_{c}=\frac{\pi}{c}$ zero-energy states for any side surface, except for [010] surface perpendicular to vector $\mathbf{M}$.

Another consequence of weak index $\mathbf{M}$ is the existence of pairs of counter-propagating zero modes ('helical modes') localized in a dislocation line, which respects mirror symmetry $\Pi_{m}$. The number of zero-mode pairs $N_{0}$ in each dislocation line is determined by its Burgers vector $\mathbf{B}$ by $N_{0}=\mathbf{B} \cdot \mathbf{M} / 2 \pi$ (ref. 23). We have performed numerical calculations that demonstrate a pair of gapless helical modes in a dislocation line along $\hat{\mathbf{c}}$ axis with Burgers vector $\mathbf{B}= \pm \hat{\mathbf{a}}$. Detailed results are presented in the Methods section.

Classification and topological invariants. To understand the topological nature of the zero-energy surface states, let us first clarify the symmetry of tight-binding model $H(\mathbf{k})$. It turns out in the mirror-reflection-symmetric $k_{c}=\frac{\pi}{c}$ plane, $H(\mathbf{k})$ has an emergent chiral symmetry, that is, $\{H(\mathbf{k}), \mathcal{C}\}=0$, where $\mathcal{C}=\sigma_{z} v_{x} \tau_{z}$. Here $\boldsymbol{\sigma}, \boldsymbol{v}$ and $\tau$ represent $J_{\text {eff }}=\frac{1}{2}$ pesudo-spin space, inter-plane and in-plane sublattice, respectively. A chiral symmetry can be understood as the combination of time-reversal symmetry and certain particle-hole symmetry ${ }^{24}$, hence switching the sign of Hamiltonian. The presence of chiral symmetry $\mathcal{C}$ enforces the energy spectrum of $H(\mathbf{k})$ to be symmetrical with respect to the zero energy. It is known that chiral symmetry can protect zero-energy surface modes ${ }^{25,26}$. On the other hand, various crystal symmetries such as mirror reflection $\Pi_{m}$ bring extra non-trivial topological properties into the system we studied. Starting from the $k_{c}=\frac{\pi}{c}$ plane with mirror reflection $\Pi_{m}=\sigma_{z} v_{x}$ and chiral symmetry $\mathcal{C}$, we can classify possible surface flat bands in the mathematical framework of K-theory ${ }^{27}$, as summarized in Table 1 (see Methods section for details).

In particular with both mirror and chiral symmetries, the classification is $\mathbb{Z} \times \mathbb{Z}$ characterized by a pair of integer topological invariants $\left(W^{+}, W^{-}\right)$. Since the Hilbert space can be decomposed into two subspaces with different mirror eigenvalues $\Pi_{m}= \pm 1$, in each subspace we can obtain a $1 \mathrm{D}$ winding number ${ }^{14}$

$$
W_{[l m n]}^{ \pm}\left(\mathbf{k}_{\|}\right)=\frac{1}{2 \pi \mathrm{i}} \int d k_{\perp} \operatorname{Tr}\left[\mathcal{C} h_{ \pm}^{-1}(\mathbf{k}) \frac{\partial}{\partial k_{\perp}} h_{ \pm}(\mathbf{k})\right],
$$

where $k_{\perp}$ is the crystal momentum along [lmn] direction and $h_{ \pm}(\mathbf{k})=H_{\mathbf{k}_{\|}}^{ \pm}\left(k_{\perp}\right)$ is the 1D Hamiltonian parametrized by [lmn] surface momentum $\mathbf{k}_{\|}$in $\Pi_{m}= \pm 1$ subspace. For both $\hat{\mathbf{b}} \hat{\mathbf{c}}$ plane ([110] surface) and $\hat{\mathbf{a}} \hat{\mathbf{c}}$ plane $\left([1 \overline{1} 0]\right.$ surface), we have $\left(W^{+}\right.$, 
a

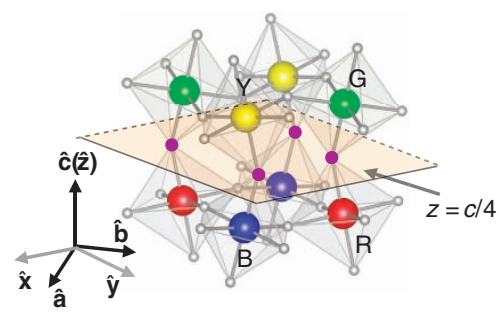

C

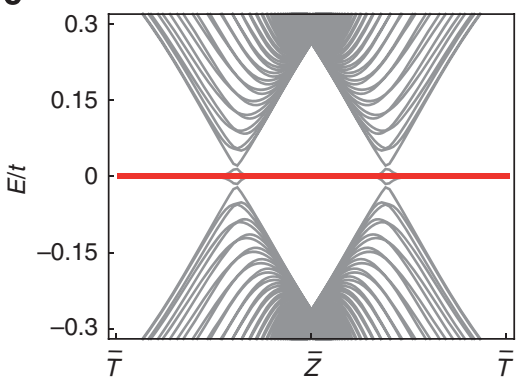

b

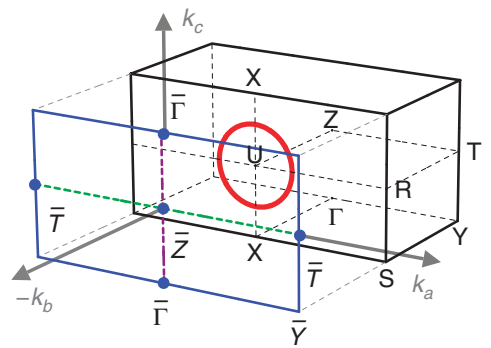

d

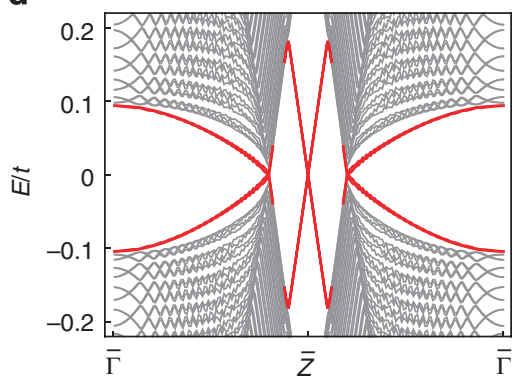

Figure 1 | Crystal structure and surface states on [110] side plane. (a) Crystal structure of $\mathrm{AlrO}_{3}$ with $\mathbf{a}$ axis along [110], $\hat{\mathbf{b}}$ axis along [110] and $\mathbf{c}$ axis along $\hat{\mathbf{z}}$ direction, respectively. The unit cell contains four Ir atoms: blue $(B)$, red $(R)$, yellow $(Y)$ and green $(G)$ represent different oxygen octahedra environment. The length of Bravais lattice unit vectors $\mathbf{a} / \hat{\mathbf{b}}$ and $\hat{\mathbf{c}}$ axis are $a / a$ and $c$, respectively. A mirror symmetry plane (coloured with light orange), mapping $z$ to $-z$, locates at $z=\frac{c}{4}$, where four oxygen atoms (purple solid circles) are within the same plane. (b) Special $k$-points in the 3D bulk BZ and 2D surface BZ for [11̄0] surface. The location of the nodal ring depict as red circles on U-R-S-X plane in 3D BZ. Slab-geometry surface spectrum (c) for [110] surface at $k_{c}=\frac{\pi}{c^{\prime}}$ plotted along the high-symmetry line (green dashed line) $\bar{T} \rightarrow \bar{Z} \rightarrow \bar{T}$, and (d) for [110] surface at $k_{a}=0$ by varying $k_{c}$ to follow $\bar{\Gamma} \rightarrow \bar{Z} \rightarrow \bar{\Gamma}$ (purple dashed line), where red lines represent surface states.

Table 1 | Classification of topological crystalline metals and possible surface states on a generic side surface parallel to $\hat{z}$-axis.

\begin{tabular}{lcccc} 
Mirror $\boldsymbol{\Pi}_{\boldsymbol{m}}$ & Chiral $\mathcal{C}$ & Classification & Topological invariants & Surface zero modes \\
\hline Yes & Yes & $\mathbb{Z} \times \mathbb{Z}$ & $\left(W^{+}, W^{-}\right)=(1,-1)$ & Yes $\left(k_{c}=\frac{\pi}{c}\right)$ \\
No & Yes & $\mathbb{Z}$ & $W^{-} \equiv W^{+}+W^{-}$ & Yes $\left(k_{c}=\frac{\pi}{c}+\delta,|\delta| \ll 1\right)$ \\
Yes & No & 0 & - & No \\
No & No & 0 & - & No \\
\hline
\end{tabular}

Note that $\left[\Pi_{m}, \mathcal{C}\right]=0 .\left(W^{+}, W^{-}\right)$is the pair of winding numbers obtained in $\Pi_{m}= \pm 1$ subspaces of block-diagonalized Hamiltonian.

$\left.W^{-}\right)=(1,-1)$. These quantized winding numbers correspond to a pair of zero modes for each surface momentum with $k_{c}=\frac{\pi}{c}$, and they cannot hybridize due to opposite mirror eigenvalues. Meanwhile, for [010] surface both $W \pm$ vanish, indicating a weak index ${ }^{22} \mathbf{M}=\hat{\mathbf{a}}+\hat{\mathbf{b}} / / \hat{\mathbf{y}}$. Intuitively, the system can be considered as a stacked array of $\hat{\mathbf{x}} \hat{\mathbf{z}}$ planes, each plane (perpendicular to $\hat{\mathbf{y}}$ axis) with a pair of mirror-protected zero-energy edge modes at $k_{c}=\frac{\pi}{c}$.

Once the mirror symmetry $\Pi_{m}$ is broken, the classification becomes $\mathbb{Z}$ characterized by one integer topological invariant: the total winding number $W \equiv W^{+}+W^{-}$associated with 1D TI in symmetry class AIII ${ }^{24,27}$. This total winding number vanishes for all surface momenta at $k_{c}=\frac{\pi}{c}$ though.

Topological metal/semimetal induced by magnetic fields. Time-reversal (TR) breaking perturbations such as a magnetic field can drive the system from the TCM phase to other metallic phases with different topological properties. In the presence of Zeeman coupling $\mu_{B} \mathbf{h} \cdot \boldsymbol{\sigma}$ introduced by magnetic field $\mathbf{h}$, clearly the chiral symmetry $\mathcal{C}$ is still preserved as long as the field is in $\hat{\mathbf{x}} \hat{\mathbf{y}}$ plane $(\mathbf{h} \perp \hat{\mathbf{z}})$. Magnetic field parallels to $\hat{\mathbf{z}}$ axis breaks chiral symmetry, which gaps the nodal ring, making the system trivial. Meanwhile, mirror symmetry $\Pi_{m}$ will be broken unless the magnetic field is along $\hat{\mathbf{z}}$ direction. Thus, our focus below will be magnetic field in the $\hat{\mathbf{x}} \hat{\mathbf{y}}$ plane. The FS topology and associated surface states with a magnetic field along different directions are listed in Table 2.

Chiral topological metal protected by chiral symmetry. Due to TR and inversion symmetry, the nodal ring in TCM always has twofold Kramers degeneracy. After applying [1힐 direction magnetic field $\mathbf{h} / / \hat{\mathbf{b}}$, the doubly degenerate nodal ring in Fig. $1 \mathbf{b}$ splits into two rings in Fig. 2a shifted along $\hat{\mathbf{c}}(\hat{\mathbf{z}})$ axis on $\mathrm{U}-\mathrm{R}-\mathrm{S}-\mathrm{X}$ plane. Although the mirror symmetry is broken by the $[1 \overline{1} 0]$ magnetic field, chiral symmetry $\mathcal{C}$ is still preserved. Therefore, the topological properties of the two nodal rings are captured by an integer winding number $W$ in the symmetry class AIII ${ }^{14}$. Consider $[1 \overline{1} 0]$ surface for instance, depending on the surface momentum $\mathbf{k}_{\|}$, the winding number $W_{\mathbf{k}_{\|}}$is plotted in Fig. 2b. It vanishes in the region where the two nodal rings (blue and red) overlap, but becomes \pm 1 in other regions within the two nodal rings.

The energy spectra on a slab geometry with open [110] surfaces are displayed in Fig. 2c,d, plotted as a function of $k_{a}$ with $k_{c}=\frac{\pi}{c}$ and $k_{c}=\frac{\pi}{c}-\delta$, respectively. There is no zero modes at $k_{c}=\frac{\pi}{c}$, 
corresponding to trivial winding number. Meanwhile, zeroenergy flat bands highlighted by red colour in Fig. 2d exist inside the nodal rings. It confirms the non-trivial topology of the bulk nodal rings with quantized winding number $W_{[1 \overline{1} 0]}= \pm 1$ shown in Fig. 2b. It turns out this chiral topological metal supports localized flat band protected by chiral symmetry on any surface, as long as its normal vector $\hat{\mathbf{n}}$ is not perpendicular to $\hat{\mathbf{b}}$ axis. Meanwhile, the two nodal rings are stable against any perturbations preserving chiral symmetry, since the winding number changes when we cross each nodal ring ${ }^{14}$.

Weyl semimetal. Once we apply a magnetic field along [110] direction (or â axis), both mirror $\left(\Pi_{m}\right)$ and n-glide $\left(\Pi_{n}\right)$ symmetries are broken. Consequently, the nodal ring is replaced by a pair of 3D Dirac nodes, appearing at momenta $\left( \pm k_{0}, \frac{\pi}{a}, \frac{\pi}{c}\right)$ along the path $\mathrm{R} \rightarrow \mathrm{U} \rightarrow \mathrm{R}$ BZ line. However, these Dirac nodes are not symmetry protected, since a sublattice potential $m v_{z}$ alternating by layers would further split each Dirac point into a pair of Weyl nodes. And this $m v_{z}$ term has the same symmetry as a Zeeman field $h_{[110]}$ along $\hat{\mathbf{a}}$ axis.

In the presence of both magnetic field $h_{[110]}$ and layeralternating potential $m v_{z}$, the system still preserves chiral symmetry $\mathcal{C}$, b-glide $\Pi_{b}$ and inversion symmetry. Two pairs of
Weyl nodes emerge at $\pm \mathbf{k}_{1}=\left( \pm k_{1}, \frac{\pi}{a}, \frac{\pi}{c}\right)$ and $\pm \mathbf{k}_{2}=$ $\left( \pm k_{2}, \frac{\pi}{a}, \frac{\pi}{c}\right)$ along $\mathrm{R} \rightarrow \mathrm{U} \rightarrow \mathrm{R}$ line in Fig. 3a. The low-energy Hamiltonian around $\mathbf{k}_{1}$ has linear dispersion along all $\mathbf{k}$ directions

$$
\begin{gathered}
H_{\text {eff }}(\delta \mathbf{k})=\mathbf{p}(\delta \mathbf{k}) \cdot \sigma \\
\mathbf{p}(\delta \mathbf{k}) \equiv\left(p_{x}, p_{y}, p_{z}\right)=\left(A_{1} \delta k_{b}-A_{2} \delta k_{c}, B_{1} \delta k_{c}, D_{1} \delta k_{a}\right),
\end{gathered}
$$

with $\delta \mathbf{k} \equiv \mathbf{k}-\mathbf{k}_{1}$. Various coefficients can be expressed in terms of the tight-binding hopping parameters (see Methods).

There exists a 'jump' for the Chern number $C$ for all occupied bands from $C=0$ to $C=1$ when $k_{a}$ crosses $k_{1}$, and similarly an opposite 'jump' from $C=1$ to $C=0$ after $k_{a}$ passes $k_{2}$. The different signs of jumps in Chern number indicate that the Weyl fermions at $\pm \mathbf{k}_{1}$ and $\pm \mathbf{k}_{2}$ have opposite topological charge +1 (blue) and -1 (red), respectively, as shown in Fig. 3a.

The surface states on [1 10$]$ surface at $k_{a}=\frac{0.7}{a}$, between the two Weyl nodes with opposite chirality, are plotted along $k_{c}$ in Fig. $3 \mathrm{~b}$. There is a single dispersing zero mode coloured with red in Fig. $3 b$, which is localized on each surface of the sample. A series of one-way-dispersing zero modes for all surface momenta between $\mathbf{k}_{1}$ and $\mathbf{k}_{2}$ form a 'chiral Fermi arc ${ }^{\text {'13,28 }}$ on [110] surface, as shown by the green lines in Fig. $3 \mathrm{a}$.

Table 2 | Various TR-breaking perturbations and consequences on the nodal ring and surface states (for example, on [110] side surface).

\begin{tabular}{lccccll} 
B field & b-glide $\boldsymbol{\Pi}_{\boldsymbol{b}}$ & n-glide $\boldsymbol{\Pi}_{\boldsymbol{n}}$ & Mirror $\boldsymbol{\Pi}_{\boldsymbol{m}}$ & Chiral $\mathcal{C}$ & Fermi surfaces & Zero-energy surface states \\
\hline$[1 \overline{1} 0]$ & No & Yes & No & Yes & Nodal rings & Flat bands at $k_{c}=\frac{\pi}{c} \pm \delta$ \\
{$[110]$} & Yes & No & No & Yes & Nodal points & Fermi arcs between Weyl points \\
{$[001]$} & No & No & Yes & No & Gapped & No
\end{tabular}

When the magnetic field is along [110] direction, the nodal ring splits into a pair of Dirac nodes along $U \rightarrow R$. On the other hand, the nodal ring is completely gapped if the magnetic field has a nonzero $\hat{\mathbf{z}}(\hat{\mathbf{c}})$ direction component.

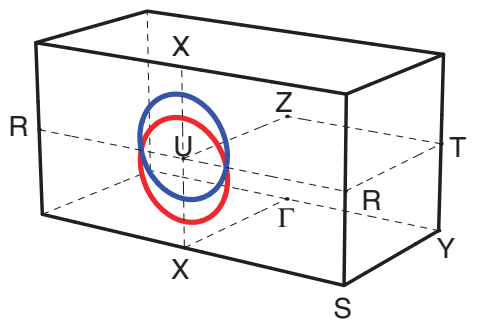

C

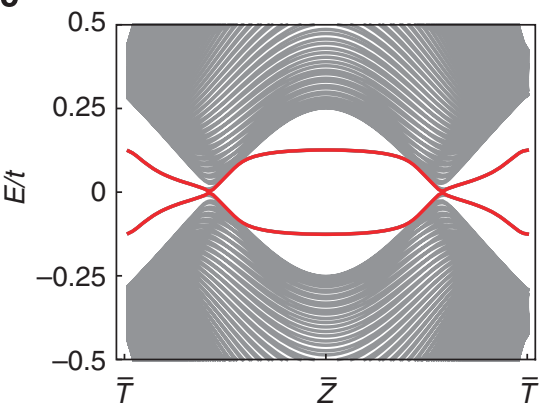

b

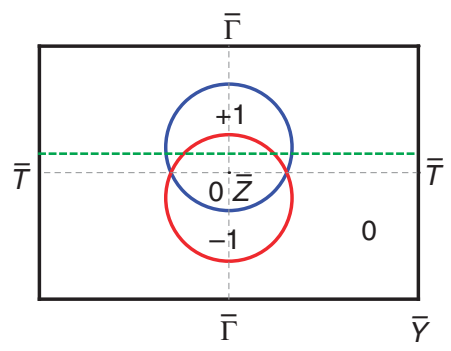

d

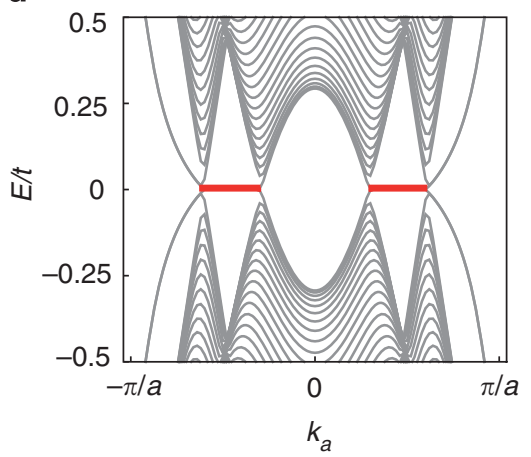

Figure 2 | Evolution of the nodal ring under various TR-breaking terms and corresponding surface modes. (a) When the magnetic field $\hat{\mathbf{h}} \| \hat{\mathbf{b}}$, a pair of nodal rings contain one ring (blue) shifted upwards along $\hat{\mathbf{c}}(\hat{\mathbf{z}})$ axis and the other one (red) shifted downwards. (b) The corresponding winding number $W_{[110]}$ distribution on $\bar{Z}-\bar{T}-\bar{Y}-\bar{\Gamma}$ plane. Slab spectrum (c) when $k_{c}=\frac{\pi}{c}$ for [11̄0] surface plotted as a function of $k_{a}$ along the high-symmetry line $\bar{T} \rightarrow \bar{Z} \rightarrow \bar{T}$, and (d) when $k_{c}=\frac{\pi}{c}+\delta$ indicated by the green dashed line in (b). 
a

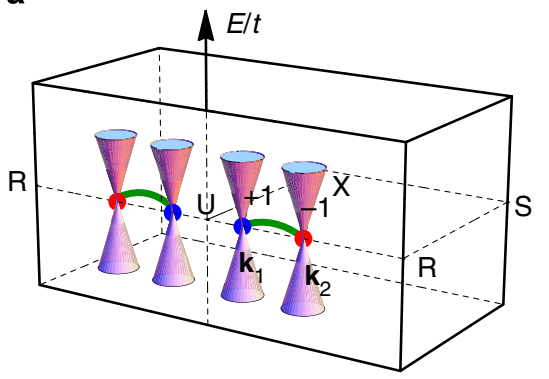

b

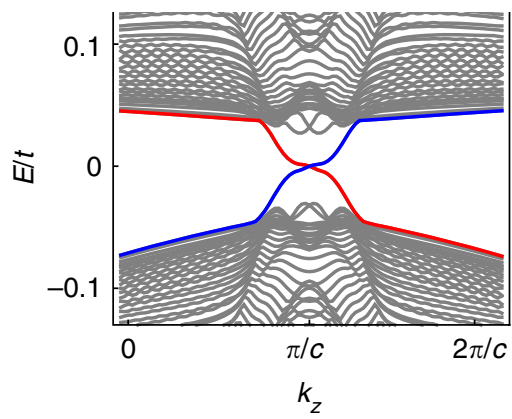

Figure 3 | Emergence of Weyl fermions on [110] side surface. (a) Two pairs of Weyl node located along the high-symmetry line $U \rightarrow R$ at $\mathbf{k}_{1}$ and $\mathbf{k}_{2}$, respectively. One of the Weyl fermion (blue) has +1 chirality but the other Weyl node (red) has opposite chirality. The Fermi arc connecting those two Weyl nodes is coloured by green. (b)The edge states spectra when $k_{a}=\frac{0.7}{a}$, which is in between two Weyl nodes plotted as a function of $k_{c}$.

\section{Discussion}

The existence of surface zero modes in our TCM phase originates from the chiral and mirror-reflection symmetry of $\mathrm{AIrO}_{3}$ with Pbnm structure. Any side surface other than [010] plane should exhibit robust zero-energy surface states independent of the details. In a generic band structure of $\mathrm{SrIrO}_{3}$, this nodal ring does not sit exactly at the Fermi level $E_{\mathrm{F}}$, but slightly below $E_{\mathrm{F}}$ (unless SOC is stronger than an atomic SOC used in the first-principle calculation), and a hole-like pocket FS occurs around $\Gamma$ point in Fig. $1 \mathrm{~b}^{19}$. In other words, the nodal ring occurs around $k_{c}=\frac{\pi}{c}$, while the small bulk Fermi pocket is located near $k_{c}=0$. Therefore, the zero-energy surface modes are well separated from the bulk FS pockets in momentum, and a momentumresolved probe is required to detect the surface states. Angleresolved photoemission spectroscopy (ARPES) would be the best tool to observe the momentum-resolved surface states shown in Fig. $1 \mathrm{c}, \mathrm{d}$ on a side plane of $\mathrm{AIrO}_{3}$. Notice that, ARPES has successfully detected the topological surface states in Dirac semimetal material ${ }^{29}$. Due to the presence of extremely small orbital overlap amplitudes between further Ir sites, the surface states acquire a slight dispersion. However, as we emphasized above, the mirror symmetry is a crucial ingredient to support such non-trivial surface states detectable by ARPES, despite the 'weak breaking' of chiral symmetry.

These surface states also contribute finite surface density of states (SDOSs) near zero energy. In contrast, the semimetallic bulk band contribution to SDOS vanishes around zero energy due to the presence of bulk nodal ring. Therefore, protected surface modes can be detected as a zero bias hump (finite SDOSs) in the $\mathrm{dI} / \mathrm{dV}$ curve of scanning tunnelling microscopy. However, in real materials, it will be difficult to separate the contribution of the surface states to SDOS from the bulk part. On the other hand, there exists protected propagating fermion modes in dislocation lines ${ }^{23}$ that preserves mirror symmetry. One advantage is that these topological helical modes are protected by mirror and chiral symmetries, and hence will not be destroyed by hybridization with bulk gapless excitations. In particular, counter-propagating gapless fermions show up in pairs in the dislocation core, and the number of gapless fermion pairs is given by $\mathbf{M} \cdot \mathbf{B} / 2 \pi$, where $\mathbf{B}$ is the Burgers vector of dislocation as shown in the Methods section. Unlike other gapless and dispersive bulk excitations that are extended in space, these helical fermion zero modes are localized near the dislocation core, which in hence can be detected by scanning tunnelling microscopy.

Since a bulk sample of $\mathrm{AIrO}_{3}$ such as $\mathrm{SrIrO}_{3}$ requires high pressure to achieve the $\mathrm{Pbnm}$ crystal structure ${ }^{30,31}$, it is desirable to grow a film of $\mathrm{AIrO}_{3}$. Recently, superlattices of atomically thin slices of $\mathrm{SrIrO}_{3}$ was made by pulsed laser deposition along [001] plane $^{32}$. This work is a first step towards possible topological phases in iridates. Furthermore, a successful growth of film along [111] plane was also reported ${ }^{33}$. Thus, growing a film of AIrO3 along [110] (or [1] 0$]$ ) is plausible. To confirm the proposed TCM, an ARPES study should be performed on a film of AIrO3 grown along [110] (or $[1 \overline{1} 0]$ ), where the mirror symmetry of Pbnm structure is kept. This analysis should reveal a flat surface band near $k_{c}=\frac{\pi}{c}$ below $E_{\mathrm{F}}$.

\section{Methods}

Symmetry operators and tight-binding Hamiltonian. The tight-binding Hamiltonian is defined in the basis of eight component spinor $\psi$, organized as ${ }^{18}$

$$
\psi \equiv\left(c_{B \uparrow}, c_{R \uparrow}, c_{Y \uparrow}, c_{G \uparrow}, c_{B \downarrow}, c_{R \downarrow}, c_{Y \downarrow}, c_{G \downarrow}\right)^{T},
$$

where $B, R, R, G$ correspond to four sublattices. We define Pauli matrices $\tau$ and $\boldsymbol{v}$ in terms of following sublattice rotations

$$
\begin{array}{ll}
B \stackrel{\tau_{x}}{\longleftrightarrow} R, \quad Y \stackrel{\tau_{x}}{\longleftrightarrow} G ; \\
B \stackrel{v_{x}}{\longleftrightarrow} Y, \quad R \stackrel{v_{x}}{\longleftrightarrow} G .
\end{array}
$$

The full space group Pbnm is generated by (see Fig. 2 in ref. 18) translations $T_{x, y, z}$ (three Bravais primitive vectors correspond to $T_{a} \equiv T_{x} T_{y}, T_{b} \equiv T_{x}^{-1} T_{y}$ and $\left.T_{c}=T_{z}^{2}\right)$ and the following three generators $\left\{\Pi_{b}, \Pi_{n}, \Pi_{m}\right\}$

$$
\begin{aligned}
& \psi_{\left(k_{a}, k_{b}, k_{c}\right)} \stackrel{\Pi_{n}}{\longrightarrow} e^{i^{\frac{k_{a}+k_{c}}{2}}} \frac{\mathrm{i}\left(\sigma_{x}-\sigma_{y}\right)}{\sqrt{2}} v_{x} \tau_{x} \cdot e^{\stackrel{\mathrm{i}^{k_{c} \nu_{z}+k_{a} \tau_{z}}}{2}} \psi_{\left(k_{a},-k_{b}, k_{c}\right)}, \\
& \psi_{\left(k_{a}, k_{b}, k_{c}\right)} \stackrel{\Pi_{b}}{\longrightarrow} e^{i \frac{k_{b}}{2}} \frac{\mathrm{i}\left(\sigma_{x}+\sigma_{y}\right)}{\sqrt{2}} \tau_{x} \cdot e^{i \frac{k_{b} \tau_{z}}{2}} \psi_{\left(-k_{a}, k_{b}, k_{c}\right)}, \\
& \psi_{\left(k_{a}, k_{b}, k_{c}\right)} \stackrel{\Pi_{m}}{\longrightarrow} \mathrm{i} \sigma_{z} v_{x} \psi_{\left(k_{a}, k_{b},-k_{c}\right)}, \\
& \psi_{\mathbf{k}} \stackrel{I}{\longrightarrow} \psi_{-\mathbf{k}} .
\end{aligned}
$$

where $\Pi_{b}$ (glide plane $\perp \hat{\mathbf{a}} \equiv \hat{\mathbf{x}}+\hat{\mathbf{y}}$ ) and $\Pi_{n}$ (glide plane $\perp \hat{\mathbf{b}} \equiv \hat{\mathbf{y}}-\hat{\mathbf{x}}$ ) represent two glide symmetries, while $\Pi_{m}$ is the mirror reflection and $\boldsymbol{\sigma}$ denotes the pseudospin subspace. $I=\Pi_{b} \Pi_{n} \Pi_{m}$ represents the inversion symmetry.

The tight-binding Hamiltonian of $\mathrm{SrIO}_{3}$ has the following form ${ }^{18}$

$$
\begin{aligned}
H_{\mathbf{k}}= & \operatorname{Re}\left(\epsilon_{\mathbf{k}}^{\mathrm{p}}\right) \tau_{x}+\operatorname{Im}\left(\epsilon_{\mathbf{k}}^{\mathrm{p}}\right) \sigma_{z} \tau_{y}+\epsilon_{\mathbf{k}}^{z} v_{x} \\
& +\operatorname{Re}\left(\epsilon_{\mathbf{k}}^{\mathrm{d}}\right) v_{x} \tau_{x}+\operatorname{Im}\left(\epsilon_{\mathbf{k}}^{\mathrm{d}}\right) v_{y} \tau_{y}+\operatorname{Re}\left(\epsilon_{\mathbf{k}}^{\mathrm{po}}\right) \sigma_{y} v_{z} \tau_{y} \\
& +\operatorname{Im}\left(\epsilon_{\mathbf{k}}^{\mathrm{po}}\right) \sigma_{x} v_{y} \tau_{z}+\operatorname{Re}\left(\epsilon_{\mathbf{k}}^{\mathrm{do}}\right) \sigma_{y} v_{x} \tau_{y}+\operatorname{Im}\left(\epsilon_{\mathbf{k}}^{\mathrm{do}}\right) \sigma_{x} v_{x} \tau_{y} \\
& +\operatorname{Re}\left(\epsilon_{\mathbf{k}}^{\mathrm{d} 1}\right) \sigma_{y} v_{y} \tau_{x}+\operatorname{Im}\left(\epsilon_{\mathbf{k}}^{\mathrm{d} 1}\right) \sigma_{x} v_{y} \tau_{x} .
\end{aligned}
$$

Here various coefficient functions have been defined in ref. 18 with additional term $\epsilon_{\mathbf{k}}^{\mathrm{d} 1}$

$$
\epsilon_{\mathbf{k}}^{\mathrm{d} 1}=t_{\mathrm{d} 1}\left(\cos \left(k_{y}\right)+\mathrm{i} \cos \left(k_{x}\right)\right) \cos \left(k_{z}\right),
$$

where $t_{1 \mathrm{D}}$ is the inter-layer next nearest-neighbour hopping due to non-vanishing rotation and tilting in local oxygen octahedra. The crystal momentum $\left(k_{a}, k_{b}, k_{c}\right)$ relates to $\left(k_{x}, k_{y}, k_{z}\right)$ simply by

$$
k_{a}=\frac{k_{x}+k_{y}}{a}, \quad k_{b}=\frac{k_{y}-k_{x}}{a}, \quad k_{c}=\frac{2 k_{z}}{c} .
$$

The basis $\psi_{\left(k_{a}, k_{b}, k_{c}\right)}$ we use here is different with the basis $\psi_{\left(k_{x}, k_{y}, k_{z}\right)}$ in ref. 18 by a unitary rotation $U_{\mathbf{k}}$

$$
\psi_{\left(k_{a}, k_{b}, k_{c}\right)} \equiv U_{\mathbf{k}}^{\dagger} \psi_{\left(k_{x}, k_{y}, k_{z}\right)}, \quad U_{\mathbf{k}}=e^{i \frac{k_{k}}{2} v_{z}} e^{\frac{i k_{k}}{2} \tau_{z}}
$$


In this basis $\psi_{\left(k_{a}, k_{b}, k_{c}\right)}$, the tight-binding Hamiltonian is related to $H_{\mathbf{k}}$ by the unitary transformation $U_{\mathbf{k}}$ in equation 6 :

$$
H(\mathbf{k}) \equiv H\left(k_{a}, k_{b}, k_{c}\right)=U_{\mathbf{k}}^{\dagger} H_{\mathbf{k}} U_{\mathbf{k}} .
$$

Clearly in $k_{c}=\frac{\pi}{c}$ (or $k_{z}=\frac{\pi}{2}$ ) plane the non-vanishing terms in $H_{\mathbf{k}}$, as in the basis $\psi_{\left(k_{x}, k_{y}, k_{z}\right)}$, contain only Pauli matrices $\left(\tau_{x}, \sigma_{z} \tau_{y}\right), v_{y} \tau_{y}$ and $\sigma_{x, y} v_{x, z} \tau_{y}$. All these Pauli matrices anti-commute with

$$
\mathcal{C}=\sigma_{z} v_{y} \tau_{z},
$$

that is, they all have chiral symmetry $\mathcal{C}$. However, in $\psi_{\left(k_{a}, k_{b}, k_{c}\right)}$ representation, the chiral symmetry $\mathcal{C}$ written as

$$
\mathcal{C}=\sigma_{z} v_{x} \tau_{z}
$$

K-theory classification procedure and topological invariants. To understand surface states on [110] surface for instance, let us focus on a 1D system $H_{\mathbf{k}}$ in momentum space parametrized by fixed momentum $k_{a} \in\left[-\frac{\pi}{a}, \frac{\pi}{a}\right)$ and $k_{c}=\frac{\pi}{c}$. Such a 1D system will have mirror reflection $\Pi_{m}=\mathrm{i} \sigma_{z} v_{x}$ in (5), as well as chiral symmetry $\mathcal{C}=\sigma_{z} v_{x} \tau_{z}$ in (12). We will classify such a gapped $1 \mathrm{D}$ system (since the bulk gap only closes at two points in $k_{c}=\frac{\pi}{c}$ plane) to see whether it has non-trivial topology, which may protect gapless surface states.

The classification of a gapped system can be understood from classifying possible symmetry-allowed mass matrices for a Dirac Hamiltonian ${ }^{27,34}$. The mathematical framework of K-theory applies to both global symmetry and certain spatial (crystal) symmetry ${ }^{35,36}$. In 1D such a Dirac Hamiltonian can be written as

$$
H_{\text {Dirac }}^{1 \mathrm{~d}}=k_{a} \gamma_{1}+m \gamma_{0}
$$

with chiral symmetry $\mathcal{C}$

$$
\left\{\mathcal{C}, \gamma_{0}\right\}=\left\{\mathcal{C}, \gamma_{1}\right\}=0
$$

mirror reflection $\Pi_{m}$

$$
\left[\Pi_{m}, \gamma_{0}\right]=\left[\Pi_{m}, \gamma_{1}\right]=0 .
$$

and $U(1)$ charge conservation $Q$

$$
\left[Q, \gamma_{0}\right]=\left[Q, \gamma_{1}\right]=0 .
$$

Any two symmetry generators among $\left(\mathcal{C}, \Pi_{m}, Q\right)$ commute with each other. Mathematically, the classification problem corresponds to the following question: given Dirac matrix $\gamma_{1}$ and symmetry matrices $\left(\mathcal{C}, \Pi_{m}, Q\right)$, what is the classifying space $\mathcal{S}$ of mass matrix $\gamma_{0}$ ? In particular, since each disconnected piece in classifying space $\mathcal{S}$ corresponds to one gapped 1D phase, how many disconnected pieces does $\mathcal{S}$ contain? Hence, the classification of gapped 1D phases with these symmetries is given by the zeroth homotopy $\pi_{0}(\mathcal{S})$.

In the K-theory classification, if there are generators that commute with all Dirac matrices and other symmetry generators, such as $U(1)$ charge symmetry generator $Q$ here satisfying $Q^{2}=(-1)^{F}$, then we say the gapped system belong to a complex class. Otherwise, it belongs to a real class. Clearly, our case belongs to the complex class because both $Q$ and $M$ commute with any other matrices.

For the $k_{c}=\pi / c$ plane on a generic [ $\left.x y 0\right]$ side surface parallel to $\hat{c}$ axis, the full symmetry group is generated by $\left(\mathcal{C}, \Pi_{m}, Q\right)$ as mentioned earlier. Note that these three symmetry generators all commute with each other while $Q^{2}=(-1)^{F}$. Together with Dirac matrix $\gamma_{1}$, they form a complex Clifford algebra $\mathrm{Cl}_{2} \times \mathrm{Cl}_{2}$ :

$$
\left\{\gamma_{1}, \mathcal{C}\right\} \times \Pi_{m} \times Q,
$$

where the generators inside the parenthesis anti-commute with each other and commute with everything outside the parenthesis. The reason we have $\mathrm{Cl}_{2} \times \mathrm{Cl}_{2}$ is because we can block-diagonalize the $k_{c}=\pi$ tight-binding Hamiltonian with respect to their $\Pi_{m}$ (mirror reflection) eigenvalue \pm 1 , and in each subspace the
Clifford algebra is $\mathrm{Cl}_{2}$ (two generator in the parenthesis). Now when we add the mass matrix $\gamma_{0}$, the complex Clifford algebra is extended to $\mathrm{Cl}_{3} \times \mathrm{Cl}_{3}$ generated by

$$
\left\{\gamma_{1}, \mathcal{C}, \gamma_{0}\right\} \times \Pi_{m} \times Q
$$

Therefore, the classifying space of mass matrix $\gamma_{0}$ is determined by the extension problem of Clifford algebra $\mathrm{Cl}_{2} \times \mathrm{Cl}_{2} \rightarrow \mathrm{Cl}_{3} \times \mathrm{Cl}_{3}$ and we label such a classifying space as $\mathcal{S}=C_{2} \times C_{2}$. The classification of gapped $1 \mathrm{D}$ phases with $\left(\mathcal{C}, \Pi_{m}, Q\right)$ symmetries is hence given by

$$
\pi_{0}\left(C_{2} \times C_{2}\right)=\pi_{0}\left(C_{2}\right) \times \pi_{0}\left(C_{2}\right)=\mathbb{Z} \times \mathbb{Z}
$$

There are two integer-valued topological invariants $\left(W^{+}, W^{-}\right)$that are $1 \mathrm{D}$ winding number ${ }^{26}$ obtained in block-diagonalized subspace with

$\Pi_{m}=\sigma_{z} v_{y}= \pm 1$

Now let us start to break $\Pi_{m}$ and $\mathcal{C}$ symmetries. When we break mirror reflection $\Pi_{m}$ (but keep $\mathcal{C}$ ), the Clifford algebra extension problem becomes $\mathrm{Cl}_{2} \rightarrow \mathrm{Cl}_{3}$

$$
\left\{\gamma_{1}, \mathcal{C}\right\} \times Q \rightarrow\left\{\gamma_{1}, \mathcal{C}, \gamma_{0}\right\} \times Q
$$

and hence the classification is $\pi_{0}\left(C_{2}\right)=\mathbb{Z}$. The topological invariant is total winding number $W=W^{+}+W^{-}$.

If we break $\mathcal{C}$ but keep $\Pi_{m}$, the extension problem is again $\left(\mathrm{Cl}_{1}\right)^{2} \rightarrow\left(\mathrm{Cl}_{2}\right)^{2}$

$$
\left\{\gamma_{1}\right\} \times \Pi_{m} \times Q \rightarrow\left\{\gamma_{1}, \gamma_{0}\right\} \times \Pi_{m} \times Q
$$

and classification is trivial since

$$
\pi_{0}\left(C_{1} \times C_{1}\right)=\pi_{0}\left(C_{1}\right) \times \pi_{0}\left(C_{1}\right)=0 \times 0=0 .
$$

If we break both $\mathcal{C}$ and $\Pi_{m}$ symmetries, our extension problem is $C l_{1} \rightarrow C l_{1}$

$$
\left\{\gamma_{1}\right\} \times Q \rightarrow\left\{\gamma_{1}, \gamma_{0}\right\} \times Q,
$$

which leads to a trivial classification $\pi_{0}\left(C_{1}\right)=0$. Therefore, we obtain the results in Table 1.

Dislocation spectrum. One implication of weak index $\mathbf{M}$ is the existence of pairs of counter-propagating zero modes localized in a dislocation line respecting mirror symmetry $\Pi_{m}$. The number of zero-mode pairs in each dislocation line is determined by its Burgers vector B: number of helical modes $=\mathbf{B} \cdot \mathbf{M} / 2 \pi$. (ref. 23)

To check such zero modes due to dislocation line, we consider pair-edge dislocations along $\hat{\mathbf{c}}$ axis, with a pair of Burgers vectors: $(\mathbf{B}=\hat{\mathbf{a}}, \mathbf{B}=-\hat{\mathbf{a}})$ perpendicular to the dislocation line. The type of dislocation core is illustrated in Fig. 4a. We consider now a 3D box with periodic boundary condition in all $(\hat{\mathbf{a}}, \hat{\mathbf{b}}, \hat{\mathbf{c}})$ directions (a 3D torus) so that the system does not have an open surface. Consider every unit cell (with four sublattices, two pseudo-spin species per site) as a lattice 'site' in Fig. 4a, then the system has hopping terms between nearestneighbour 'sites' following the tight-binding Hamiltonian in equation (6). $\hat{\mathbf{c}}$ direction is still translational invariant and $k_{\mathrm{z}}$ remains a good quantum number, but in $\hat{\mathbf{a}} \hat{\mathbf{b}}$ plane translation symmetry is broken by the dislocation. The dislocation spectrum is obtained when there are 39 sites along $\hat{\mathbf{a}}$ axis and 15 sites along $\mathbf{b}$ axis. The location of dislocation with Burgers vector $\mathbf{B}=\hat{\mathbf{a}}$ is at $(4,6)$, which means 4th site along $\mathbf{A}$ axis and 6th site along $\mathbf{b}$ axis. And the position of dislocation with Burgers vector $\mathbf{B}=-\hat{\mathbf{a}}$ is at $(24,12)$. The dislocation spectrum has displayed in Fig. $4 \mathrm{~b}$ highlighted by red colour. It shows two pairs of gapless helical modes localized on each dislocation cores.

Effective Hamiltonian of Weyl fermion. By projecting the states around the Weyl node, the two-band Hamiltonian with linear Weyl fermion form can be obtained after adding $m v_{z}$, which breaks mirror symmetry and magnetic field $h_{[110]}\left(\sigma_{x}+\sigma_{y}\right)$, which breaks TR symmetry to $H(\mathbf{k})$ in equation (6). The followings are the coefficients for the effective Hamiltonian describes the Weyl fermion at $\mathbf{k}_{1}$ of a

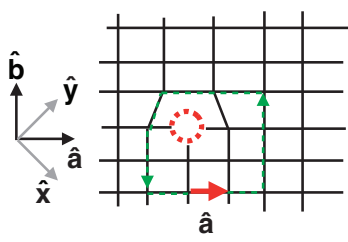

b

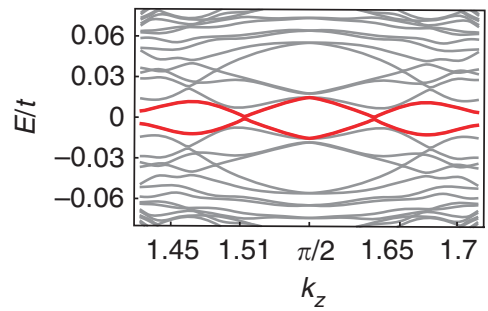

C

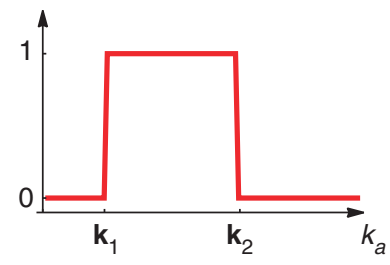

Figure 4 | Disloction spectrum and Chern number. (a) Top view of an edge dislocation with Burgers vector â (coloured by red arrow), whose dislocation line is along $\hat{\mathbf{c}}$ axis. Dashed green line denotes a trajectory around the dislocation core (red circle). Clearly when we go around the dislocation once, we need an extra translation by the Burgers vector (the red arrow), equal to Bravais primitive vector â in this case) to return to the starting point. (b) The dislocation spectrum for system size $39 \times 15$ contains two pairs of gapless helical modes (highlighted with red colour) localized at each dislocation line. (c) Chern number as a function of $k_{a}$ for all occupied bands. Opposite Chern number jump indicates that the Weyl fermion at $\mathbf{k}_{1}$ and $\mathbf{k}_{2}$ has different chirality. 
equation (2) presented in the main text:

$$
\begin{aligned}
A_{1} & =\frac{2 t_{\mathrm{p}} t_{\mathrm{d}}^{\mathrm{o}} \sin ^{2}\left(k_{1}\right)}{\left(t_{2 \mathrm{p}}^{\mathrm{o}}-t_{1 \mathrm{p}}^{\mathrm{o}}\right) \cos \left(k_{1}\right)}, \\
A_{2} & =\frac{(\sqrt{2} h-m) t_{\mathrm{z}}^{\mathrm{o}}}{\sqrt{2}\left(t_{2 \mathrm{p}}^{\mathrm{o}}-t_{1 \mathrm{p}}^{\mathrm{o}}\right) \cos \left(k_{1}\right)}, \\
B_{1} & =\frac{t_{\mathrm{d} 1}(m-\sqrt{2} h)}{\sqrt{2}\left(t_{2 \mathrm{p}}^{\mathrm{o}}-t_{1 \mathrm{p}}^{\mathrm{o}}\right)}, \\
D_{1} & =\frac{\left[\left(t_{2 \mathrm{p}}^{\mathrm{o}}-t_{1 \mathrm{p}}^{\mathrm{o}}\right)^{2}-\left(t_{d}^{\mathrm{o}}\right)^{2}\right] \sin \left(k_{1}\right)}{t_{2 \mathrm{p}}^{\mathrm{o}}-t_{1 \mathrm{p}}^{\mathrm{o}}},
\end{aligned}
$$

where $t_{\mathrm{p}}, t_{2 \mathrm{p}}^{\mathrm{o}}, t_{1 \mathrm{p}}^{\mathrm{o}}, t_{\mathrm{d}}^{\mathrm{o}}, m, t_{\mathrm{d} 1}, h \equiv h_{[110]}$ are the coefficients in tight-binding Hamiltonian. The Chern number $C$ for all occupied bands as a function of crystal momentum along $\mathrm{U} \rightarrow \mathrm{R}$ (or $k_{a}$ ) is shown in Fig. 4c.

\section{References}

1. Kane, C. L. \& Mele, E. J. Quantum spin hall effect in graphene. Phys. Rev. Lett. 95, 226801 (2005).

2. Kane, C. L. \& Mele, E. J. $Z_{2}$ topological order and the quantum spin hall effect. Phys. Rev. Lett. 95, 146802 (2005).

3. Bernevig, B. A., Hughes, T. L. \& Zhang, S.-C. Quantum spin hall effect and topological phase transition in HgTe quantum wells. Science 314, 1757-1761 (2006).

4. Konig, M. et al. Quantum spin hall insulator state in $\mathrm{HgTe}$ quantum wells. Science 318, 766-770 (2007).

5. Fu, L., Kane, C. L. \& Mele, E. J. Topological insulators in three dimensions. Phys. Rev. Lett. 98, 106803 (2007).

6. Moore, J. E. \& Balents, L. Topological invariants of time-reversal-invariant band structures. Phys. Rev. B 75, 121306 (2007)

7. Roy, R. Topological phases and the quantum spin hall effect in three dimensions. Phys. Rev. B 79, 195322 (2009).

8. Hsieh, D. et al. A Topological Dirac insulator in a quantum spin hall phase. Nature 452, 970-974 (2008).

9. Hsieh, D. et al. Observation of unconventional quantum spin textures in topological insulators. Science 323, 919-922 (2009).

10. Fu, L. Topological crystalline insulators. Phys. Rev. Lett. 106, 106802 (2011).

11. Kargarian, M. \& Fiete, G. A. Topological crystalline insulators in transition metal oxides. Phys. Rev. Lett. 110, 156403 (2013).

12. Hsieh, T. H., Liu, J. \& Fu, L. Topological crystalline insulators and Dirac octets in antiperovskites. Phys. Rev. B 90, 081112 (2014).

13. Wan, X., Turner, A. M., Vishwanath, A. \& Savrasov, S. Y. Topological semimetal and fermi-arc surface states in the electronic structure of pyrochlore iridates. Phys. Rev. B 83, 205101 (2011).

14. Matsuura, S., Chang, P.-Y., Schnyder, A. P. \& Ryu, S. Protected boundary states in gapless topological phases. New J. Phys. 15, 065001 (2013).

15. Witczak-Krempa, W., Chen, G., Kim, Y. B. \& Balents, L. Correlated quantum phenomena in the strong spin-orbit regime. Annu. Rev. Condens. Matter Phys. 5, 57-82 (2014).

16. Kargarian, M., Wen, J. \& Fiete, G. A. Competing exotic topological insulator phases in transition-metal oxides on the pyrochlore lattice with distortion. Phys. Rev. B 83, 165112 (2011).

17. Yang, B.-J. \& Nagaosa, N. Emergent Topological Phenomena in Thin Films of Pyrochlore Iridates. Phys. Rev. Lett. 112, 246402 (2014).

18. Carter, J.-M., Shankar, V. V., Zeb, M. A. \& Kee, H.-Y. Semimetal and topological insulator in perovskite iridates. Phys. Rev. B 85, 115105 (2012).

19. Zeb, M. A. \& Kee, H.-Y. Interplay between spin-orbit coupling and hubbard interaction in $\mathrm{SrIro}_{3}$ and related Pbnm perovskite oxides. Phys. Rev. B 86, 085149 (2012).
20. Cao, G. et al. Non-Fermi-liquid behavior in nearly ferromagnetic $\mathrm{SrIrO}_{3}$ single crystals. Phys. Rev. B 76, 100402 (2007).

21. Moon, S. J. et al. Dimensionality-controlled insulator-metal transition and correlated metallic state in $5 \mathrm{~d}$ transition metal oxides $\mathrm{Sr}_{n+1} \mathrm{Ir}_{n} \mathrm{O} 3 n+1$ $(\mathrm{n}=1,2$, and $\infty)$. Phys. Rev. Lett. 101, 226402 (2008).

22. Ran, Y. Weak indices and dislocations in general topological band structures. Preprint at http://arxiv.org/abs/1006.5454 (2010).

23. Ran, Y., Zhang, Y. \& Vishwanath, A. One-dimensional topologically protected modes in topological insulators with lattice dislocations. Nat. Phys 5, 298-303 (2009).

24. Schnyder, A. P., Ryu, S., Furusaki, A. \& Ludwig, A. W. W. Classification of topological insulators and superconductors in three spatial dimensions. Phys. Rev. B 78, 195125 (2008).

25. Heeger, A. J. et al. Solitons in conducting polymers. Rev. Mod. Phys. 60, 781-850 (1988).

26. Schnyder, A. P. \& Ryu, S. Topological phases and surface flat bands in superconductors without inversion symmetry. Phys. Rev. B 84, 060504 (2011)

27. Kitaev, A. Periodic table for topological insulators and superconductors. AIP Conf. Proc. 1134, 22-30 (2009).

28. Yang, K.-Y., Lu, Y.-M. \& Ran, Y. Quantum hall effects in a weyl semimetal: Possible application in pyrochlore iridates. Phys. Rev. B 84, 075129 (2011).

29. Yi, H. et al. Evidence of Topological Surface State in Three-Dimensional Dirac Semimetal $\mathrm{Cd}_{3} \mathrm{As}_{2}$. Sci. Rep. 4, 6106 (2014).

30. Longo, J., Kafalas, J. \& Arnott, R. Structure and properties of the high and low pressure forms of $\mathrm{SrIrO}_{3}$. J. Solid State Chem. 3, 174-179 (1971).

31. Zhao, J. G. et al. High-pressure synthesis of orthorhombic $\mathrm{SrIrO}_{3}$ perovskite and its positive magnetoresistance. J. Appl. Phys. 103, 103706 (2008).

32. Matsuno, J. et al. Engineering spin-orbital magnetic insulator by tailoring superlattices. Preprint at http://arxiv.org/abs/1401.1066 (2014).

33. Hirai, D., Matsuno, J. \& Takagi, H. Fabrication of (111)-oriented $\mathrm{Ca}_{0.5} \mathrm{Sr}_{0.5} \mathrm{IrO}_{3}$ $\mathrm{SrTiO}_{3}$ superlattices-A designed playground for honeycomb physics. APL Mat. 3, 041508 (2015).

34. Wen, X.-G. Symmetry-protected topological phases in noninteracting fermion systems. Phys. Rev. B 85, 085103 (2012).

35. Morimoto, T. \& Furusaki, A. Topological classification with additional symmetries from Clifford algebras. Phys. Rev. B 88, 125129 (2013).

36. Lu, Y.-M. \& Lee, D.-H. Inversion symmetry protected topological insulators and superconductors. Preprint at http://arxiv.org/abs/1403.5558 (2014).

\section{Acknowledgements}

This work is supported by Natural Science and Engineering Research Council of Canada (NSERC), Center for Quantum Materials at the University of Toronto (Y.C. and H.-Y.K.) and Office of BES, Materials Sciences Division of the U.S. DOE under contract No. DE-AC02-05CH11231 (Y.-M.L.). H.-Y.K. thanks S. Ryu for informing topology of gapless superconductors in Ref. 36. Y.-M.L. and H.-Y.K. acknowledge the hospitality of the Aspen Cetner for Physics supported by National Science Foundation Grant No. PHYS-1066293 where a part of this work was carried out.

\section{Author contributions}

All authors performed the theoretical calculations, discussed the results and wrote the manuscript. H.-Y.K. planned and supervised the project.

\section{Additional information}

Competing financial interests: The authors declare no competing financial interests.

Reprints and permission information is available online at http://npg.nature.com/ reprintsandpermissions/

How to cite this article: Chen, Y. et al. Topological crystalline metal in orthorhombic perovskite iridates. Nat. Commun. 6:6593 doi: 10.1038/ncomms7593 (2015). 